\title{
Fisioterapia complexa descongestiva no tratamento do linfedema de membro superior pós-mastectomia radical: revisão de literatura
}

\author{
Complex decongestive physiotherapy in the treatment of upper limb lymphedema after \\ radical mastectomy
}

Fisioterapia descongestiva compleja en el tratamiento del linfedema del miembro superior tras una mastectomía radical

Alaiana Marinho Franco ${ }^{1,2 \star}$, Paula Monteiro Fonteneles ${ }^{2}$, Aurileni Gomes Canto ${ }^{1}$, Ana Rute Alencar ${ }^{1}$, Larissa Marinho Franco ${ }^{3}$, Thayz Gadelha de Paula Moreira ${ }^{2}$, Armando Rodrigues de Alencar Santos², Nathálya Caitano Silva².

\section{RESUMO}

Objetivo: Analisar os benefícios e a aplicabilidade da fisioterapia complexa descongestiva no tratamento das disfunções ocasionadas pela presença do linfedema de membro superior após a cirurgia de câncer de mama (Mastectomia radical). Revisão Bibliográfica: O câncer de mama tem se destacado nas últimas décadas com grande incidência no sexo feminino, sendo a mastectomia radical uma das abordagens de tratamento, essa pode gerar complicações tardias, o linfedema de membro superior. O objetivo do tratamento vai além de razões estéticas, busca evitar infecções repetidas, progressão do aumento do volume do membro, alterações tróficas da pele e invalidez. A fisioterapia complexa descongestiva é uma técnica bastante recomendada essa é composta pela drenagem linfática manual, vestuário de compressão e cuidados com a pele, melhorando a condição patológica do linfedema, sendo reconhecida como padrão ouro de tratamento devido aos benefícios. Considerações finais: $O$ presente estudo demonstrou a eficácia da Fisioterapia Complexa Descongestiva na melhora da qualidade de vida de mulheres portadoras de linfedema de membro superior, onde as técnicas de acordo com as evidências apresentadas evoluem de forma significativa a qualidade de vida das pacientes acometidas.

Palavras-chave: Fisioterapia, Linfedema, Mastectomia radical.

\begin{abstract}
Objective: To analyze the benefits and applicability of complex decongestive physiotherapy in the treatment of disorders caused by the presence of upper limb lymphedema after breast cancer surgery (radical mastectomy). Bibliographical Review: Breast cancer has been highlighted in recent decades with great incidence in women, with radical mastectomy being one of the approaches to treatment, which can generate late complications, the upper limb lymphedema. The objective of the treatment goes beyond aesthetic reasons, it seeks to avoid repeated infections, progression of limb volume increase, trophic skin alterations and disability. The complex decongestive physiotherapy is a highly recommended technique which is composed of manual lymphatic drainage, compression clothing and skin care, improving the pathological condition of the lymphedema, being recognized as the gold standard of treatment due to the benefits. Final considerations: The present study demonstrated the effectiveness of Complex Decongestive Physiotherapy in improving the quality of life of women with upper limb lymphedema, where the techniques according to the evidence presented evolve significantly the quality of life of affected patients.
\end{abstract}

Keywords: Physiotherapy, Lymphedema, Mastectomy radical.

${ }^{1}$ Centro Universitário Metropolitana de Marabá, Marabá - PA. *E-mail: alaianamarinhofranco@hotmail.com

2 Unidade de Ensino Superior do Sul do Maranhão (UNISULMA), Imperatriz - MA.

${ }^{3}$ Centro Universitário Pitágoras de Marabá, Marabá - PA. 


\section{RESUMEN}

Objetivo: Analizar los beneficios y la aplicabilidad de la fisioterapia descongestiva compleja en el tratamiento de los trastornos causados por la presencia de linfedema en las extremidades superiores después de la cirugía del cáncer de mama (mastectomía radical). Revisión bibliográfica: El cáncer de mama se ha destacado en los últimos decenios con gran incidencia en las mujeres, siendo la mastectomía radical uno de los enfoques de tratamiento, que puede generar complicaciones tardías, el linfedema del miembro superior. El objetivo del tratamiento va más allá de las razones estéticas, busca evitar las infecciones repetidas, la progresión del aumento del volumen del miembro, las alteraciones tróficas de la piel y la discapacidad. La fisioterapia descongestiva compleja es una técnica muy recomendada que se compone de drenaje linfático manual, ropa de compresión y cuidado de la piel, mejorando la condición patológica del linfedema, siendo reconocida como el estándar de oro del tratamiento debido a sus beneficios. Consideraciones finales: Este estudio demostró la eficacia de la Fisioterapia Descongestiva Compleja en la mejora de la calidad de vida de las mujeres con linfedema de miembros superiores, donde las técnicas, según las pruebas presentadas, hacen evolucionar significativamente la calidad de vida de los pacientes afectados.

Palabras clave: Fisioterapia, Linfedema, Mastectomía radica.

\section{INTRODUÇÃO}

De acordo com os autores De Sousa AS e Neves PO (2016) é discorrido que o câncer que acomete a mama é causado por um tumor maligno na qual se desenvolve por alterações que podem ser genéticas nas células mamarias, onde passam a se dividir de forma descontrola, ocorrendo o crescimento anormal, podendo ocorrer tanto nos ductos mamário ou nos glóbulos mamários.

O carcinoma é um nódulo palpável, que quando surge na mama costuma ser doloroso ou não, sendo detectado no exame clínico, alguns nódulos podem se desenvolver nas axilas ou se envolver na caixa torácica, na forma clínica se apresenta como linfonodos axilares.

A condição patológica do câncer de mama tem se destacado com uma grande incidência nas últimas décadas no sexo feminino, na qual a mastectomia que é uma cirurgia de grande porte para a retirada da mama de forma radical ou parcial traz consigo inúmeras sequelas, devido a estrutura da mama feminina ser composta por diversas estruturas e se classifica como uma área complexa, sendo uma das maiores consequências o linfedema de membro superior (CORÍNTIO MN, 2015; CENDRON SW, et al., 2015).

Contudo o câncer de mama pode ser considerado como a principal causa de mortalidade entre o público feminino no Brasil, sendo uma das neoplasias mais temidas por elas, tanto pela alta incidência como a própria distorção de imagem.

O tratamento do câncer de mama envolve cirurgias como mastectomia radical, conservadora e linfadenectomia; quimioterapia; radioterapia e a hormonioterapia. Podendo ser administradas mais de uma terapêutica, caso necessário, sendo observado as características individuais biológicas de cada paciente e do tratamento, tendo como objetivo o controle da doença (PAIVA DMF, et al., 2011).

O tratamento do câncer de mama abrange a forma multidisciplinar e envolvendo a cirurgia, a quimioterapia, a radioterapia e tratamentos farmacológicos, na qual pode ser administrada uma ou várias terapêuticas, tendo como objetivo 18 sempre o controle da doença e à melhora da qualidade de vida (QV), após o tratamento (NGOMANE AY, et al., 2014).

Em sua pesquisa Cendron SW, et al. (2015) descreve que o câncer de mama se caracteriza como o maior índice de óbito e incidência na população feminina. Independente da conduta cirúrgica realizada no tratamento, inúmeras complicações podem surgir no período pós-operatório, ocasionando sintomas dolorosos e incapacitantes funcional, gerando complicações no processo de recuperação da paciente. Entre as alterações frequentes, o linfedema aparece como uma das complicações após a cirurgia da mama, sendo em grande parte a mais comum, devido à dissecação dos linfonodos axilares 
O tratamento é direcionado de acordo com as características do tumor, da paciente e da fase na qual a doença é diagnosticada. Quando identificado essas características opta-se pela melhor modalidade de tratamento, podendo ele ser conservador (setorectomia, tumorectomia e quadrantectomia) ou pela abordagem de tratamento radical (mastectomia simples, radical ou radical modificada) e ainda, de acordo com a evolução pode determina-se a necessidade do uso de radioterapia ou terapia sistêmica - quimioterapia e hormonioterapia (ALBUQUERQUE VT, et al., 2013).

Logo após o procedimento cirúrgico e excisão ou radiação das cadeias linfáticas regionais, a paciente submetida pode apresentar, o linfedema de membro superior, entre outras complicações, sendo sua incidência dependente de variáveis como extensão da cirurgia axilar, recorrência de câncer nos linfonodos, presença de obesidade ou paciente submetida a radioterapia (LEAL NFBS, et al, 2009).

As terapias adjuvantes assim como as próprias para o tratamento do câncer de mama, podem originar em algumas complicações físicas, sendo as mais comuns: infecção, necrose de pele por falta de circulação, presença de seroma, aderência e deiscência cicatriciais, lesão de nervos motor ou sensitivo (NASCIMENTO SL, et al., 2012).

Existem também as complicações físicas, sendo frequentemente encontradas após o tratamento, são elas: a fadiga, redução da massa muscular e em contrapartida o aumento do percentual de gordura corporal, redução da capacidade cardiopulmonar e diminuição global de força corporal, assim também como o linfedema (ALBUQUERQUE VT, et al., 2013).

A fisiopatologia do linfedema como complicação do pós-operatório de mastectomia radical ainda não é completamente compreendida. A literatura revela que existem várias teorias. Fisiopatologicamente o linfedema pode ser caracterizado pela deficiência ou diminuição de transporte de linfa pelo sistema linfático, sendo assim abaixo do fisiológico; na qual esse transporte é essencial para absorver a linfa e toxinas sanguíneo que se acumula no meio intersticial (REZENDE LF, et al., 2010).

A origem do linfedema é devido a retirada dos gânglios linfáticos na região axilar, que geralmente é acometida, na qual, sua incidência dependente de variáveis como extensão da cirurgia axilar, presença de obesidade ou paciente submetida a radioterapia. A presença do linfedema pode vim a ser um agravo crônico, que, mesmo não sendo curável, pode ser tratado e acompanhado, com condutas que podem prevenir suas complicações (SOARES HPS, et al., 2016; LEAL NFBS, et al., 2009; BRANDÃO ML et al., 2020.).

O tratamento do linfedema objetiva além de razões estéticas, pois de acordo com a Sociedade Internacional de Linfologia, a falta de controle da presença de excesso de linfa pode gerar infecções repetidas como a celulite e linfangite, assim como a progressão do aumento do volume e tamanho do membro, alterações tróficas da pele, invalidez e perca da mobilidade e, em algumas situações o desenvolvimento de um angiosarcoma altamente letal.

A técnica considerada padrão ouro e que mostra grande eficácia no tratamento do linfedema, é a terapia complexa descongestiva (TCD), na qual consiste na associação de várias técnicas associadas a favor da melhora do paciente (SOARES HPS, et al., 2016).

Conhecida mundialmente como Complex Physical Therapy (CPT), consiste na combinação de quatro técnicas principais atuando em conjunto, sendo estas a drenagem linfática manual, cuidados com a pele, enfaixamento compressivo e exercícios terapêuticos (SANTOS RO e LUZ KRG, 2017).

A fisioterapia complexa descongestiva engloba uma série de medidas, na qual inclui-se drenagem linfática manual, o vestuário de compressão, uso de bandagens elásticas, higiene da pele e exercícios linfocinéticos terapêuticos.

Ela é dividida obrigatoriamente em duas fases: a primeira em uma fase intensa de tratamento diário, a qual é aplicada em um período que dura de duas a quatro semanas, onde só é finalizada quando atingir o máximo de redução do linfedema (inchaço); e a segunda fase do tratamento se denomina de manutenção, em que especialmente a terapia de compressão é mantida continuamente, para garantir os efeitos positivos alcançados durante a primeira fase (LUZ ND e LIMA ACG, 2011; BRANDÃO ML, et al., 2020). 
A presente pesquisa bibliográfica objetivou evidenciar a eficácia da terapia complexa descongestiva no tratamento de complicações após a cirurgia de mastectomia, através do levantamento literário de estudos que reuniam conhecimentos e pesquisas acerca do tema de forma objetiva e clara.

\section{REVISÃO BIBLIOGRÁFICA}

Foi descrito que dentre os tipos de mastectomi4a estão à mastectomia radical, na qual se trata da retirada do tumor e das glândulas mamárias, dos músculos peitorais, da pele adjacente e dos linfonodos axilares próximo a mama; a Mastectomia radical modificada de Patey é um outro método onde é retirado o músculo peitoral menor, a pele e os linfonodos axilares; Mastectomia Radical de Halstedremoção é retirado os musculos peitoral maior e peitoral menor, a mama, incluindo pele, e três níveis de linfonodos axilares a mastectomia radical modificada de Madden onde a mama, a pele e os linfonodos axilares são retirados.

Apesar de que a abordagem cirúrgica seja o melhor tratamento com relação à doença em estádio avançado, também é a mais invasiva e de pior prognostico no pós-operatório pode levar a alterações na qualidade vida das mulheres, incluindo prejuízos psicológicos e físicos, precoces ou tardios (NASCIMENTO SL, et al., 2012).

A cirurgia de mastectomia pode originar uma série de complicações físicas e motoras como: infecção, presença de linfedema, limitação da capacidade funcional do braço e do ombro, dor frequente e parestesia, podendo assim colocar em risco o desempenho das atividades de vida diária e dos papéis da mulher mastectomizada. Essas complicações como a restrição na funcionalidade dos movimentos do ombro, linfedema e presença de alteração de sensibilidade, estão fortemente relacionadas com a associação da técnica cirúrgica executada, onde ocorre dissecção de linfonodos axilares após radioterapia.

A mastectomia está associada a sequelas e complicações em até $70 \%$ dos casos abordados, afetando de forma negativa a qualidade de vida das pacientes. Além das sequelas relacionadas com a retirada dos linfonodos axilares durante a cirurgia, prejudicando a capacidade de transporte do sistema linfático (REZENDE LF, et al., 2010; NOGUEIRA PVG, 2018).

Os avanços científicos do câncer e mama possibilitam uma nova perspectiva na qualidade de vida e sobrevida dos pacientes, em contrapartida ao mesmo tempo são responsáveis por diversas complicações precoces ou tardias oriundos das intervenções. Tendo um grande destaque nas complicações físicas do momento pós-cirúrgico é o linfedema de membro superior, condição que causa incapacidade crônica e incurável (DE ROMA MAM, et al., 2016).

A fisioterapia atua efetivamente no tratamento do câncer de mama minimizando assim ou prevenindo as sequelas provenientes do tratamento, favorecendo o retorno às atividades de vida diária e uma melhor qualidade de vida. Fornecendo orientações para prevenção e tratamento das complicações, sendo por meio de cuidados com a pele, realização de exercícios funcionais e autodrenagem para que os pacientes realizem em casa, essas são opções que fazem parte das estratégias fisioterapêuticas que podem dribla as dificuldades econômicas e de deslocamento das pacientes, onde muitas enfrentam para comparecerem às sessões de fisioterapia, além disso, servem para reduzir os serviços nessa área (NGOMANE AY, et al., 2014).

Santiago GS, et al. (2016) relata que: A presença do linfedema em pacientes de pós mastectomia é em torno de $20 \%$ a $30 \%$ com uma taxa de prevalência de $15 \%$ a $30 \%$ das pacientes. A presença do linfedema pode vim a ser um agravo crônico, que, mesmo não sendo curável, pode ser tratado e acompanhado, com condutas que podem prevenir suas complicações, sendo que uma vez instalado e não tratado da forma correta, pode evoluir e interferir de forma negativa na qualidade de vida das pacientes afetadas, proporcionando, além de problemas físicos, complicações psíquicas e sociais, assim como nas atividades de vida diária (SOARES HPS, et al., 2016).

O aumento de tamanho do membro devido ao volume do membro pode gerar desfiguração da imagem corporal feminina, assim como aumentar da limitação física e psicológica da paciente, além de promover significativo prejuízo para a função. Apesar da existência de vários tratamentos satisfatórios, ainda existe a falta de conhecimento sobre prevenção e a cura do linfedema, onde faz com que os profissionais de saúde continuem em busca de melhores resultados (REZENDE LF, et al., 2010). 
De Roma MAM, et al. (2016) diz que o linfedema é uma das complicações do pós operatório de câncer de mama, sendo de característica crônica, comum aos pacientes tratados cirurgicamente devido aos danos produzidos no sistema linfático.

Sua presença e prevalência causa deformidade no membro, restrição e incapacidade física e funcional podendo provocar um maior índice de estresse, depressão e sofrimento emocional nas pacientes acometidas. Para o adequado tratamento e manejo do linfedema é fundamental que se busque formas de atuação sobre o sistema linfático, favorecendo dessa forma a diminuição da progressão e severidade da presença de edema.

O tratamento do linfedema objetiva além de razões estéticas, pois de acordo com a Sociedade Internacional de Linfologia, a falta de controle da presença de excesso de linfa pode gerar infecções repetidas como a celulites e linfangites, assim como a progressão do aumento do volume e tamanho do membro, alterações tróficas da pele, invalidez e perca da mobilidade e, em algumas situações o desenvolvimento de um angiosarcoma altamente letal.

Devido não ser possível obter a cura desta sequela, a redução do volume de liquido dos membros acometidos, minimiza a sobrecarga articular e reduz a incidência de dor, proporcionando a melhora na realização das atividades de vida diária (SOARES HPS, et al., 2016).

A fisioterapia complexa descongestiva engloba uma série de medidas, na qual inclui-se drenagem linfática manual, o vestuário de compressão, uso de bandagens elásticas, higiene da pele e exercícios linfocineticos terapêuticos. Ela é dividida obrigatoriamente em duas fases: a primeira em uma fase intensiva de tratamento diário, a qual é aplicada em um período que dura de duas a quatro semanas, onde só é finalizada quando atingir o máximo de redução do linfedema (inchaço); e a segunda fase do tratamento se denomina de manutenção, em que especialmente a terapia de compressão é mantida continuamente, para garantir os efeitos positivos alcançados durante a primeira fase (LUZ ND e LIMA ACG, 2011).

De acordo com Soares HPS, et al. (2016) a técnica considerada padrão ouro e que mostra grande eficácia no tratamento do linfedema, é a terapia complexa descongestiva, na qual consiste na associação de várias técnicas associadas a favor da melhora do paciente, sendo conhecida mundialmente como CPT.

Contudo Cendron SW, et al. (2015) descreve que no tratamento e melhora do linfedema, a fisioterapia vem ocupando um lugar de destaque, na qual pode ser realizada em duas fases de tratamento: A primeira é a fase intensiva, composta pela (FCD) fisioterapia complexa descongestiva, na qual a técnica combina drenagem linfática manual (DLM) com outros procedimentos (enfaixamento compressivo funcional (ECF), bandagem elástica funcional (kinesiotape), contenção elástica, compressão pneumática intermitente (CPI) associados aos exercícios cinesioterapêuticos, assim como os cuidados com a pele (limpeza e hidratação) e 9 cuidados na vida diária. A segunda fase é a de manutenção, onde os recursos mais aplicados consistem nos cuidados com a pele, na automassagem linfática, exercícios funcionais, uso de contenção elástica.

Nessa última fase, a paciente deve ser informada e conscientizada da cronicidade da patologia, da obrigatoriedade de controles periódicos e da necessidade constante da contenção elástica e cuidados, pois a manutenção e prevenção de complicações vai depender dos cuidados do paciente (LUZ ND e LIMA ACG, 2011).

Um dos principais métodos na abordagem de FCD é drenagem linfática manual (DLM), técnica de massagem com manobras lentas, movimentos rítmicos e suaves que manipulam a superfície da pele e visam os caminhos anatômicos do sistema linfático do corpo, tem como objetivo drenar o excesso de líquido presente no interstício, nos tecidos e dentro dos vasos linfáticos, estimula pequenos capilares inativos na região; e pode aumentar a motricidade da unidade linfática (linfangion), também pode dissolver fibroses linfostáticas que estão presentes em linfedemas mais severos (SANTOS DAD, et al., 2010; LUZ ND e LIMA ACG, 2011).

A bandagem elástica também tem sua contribuição no tratamento do linfedema, possui finalidade de melhorar o fluxo linfático através da produção de diferentes estímulos e pressões na pele, onde favorece o bombeamento da linfa para as regiões com menos pressão oferecendo a vantagem de permanecer aderida na pele por dias, promovendo maior conforto quando o membro permanece em repouso, favorecendo ainda a aderência da terapêutica pelos pacientes (CENDRON SW, et al., 2015). 
Santos RO e Luz KRG (2017) relatam que exercícios fisioterapêuticos e enfaixamento compressivo podem promover reações fisiológicas benéficas para a redução do linfedema e suas consequências, mediante ações preventivas e exercícios cinesioterapicos que visam restaurar a integridade articular e mobilidade cinética funcional, na qual venha favorecer a reabsorção da linfa pelo sistema linfático, proporcionando assim os efeitos adversos a patologia e fazendo com que as mulheres mastectomizadas tenham menor incapacidade ao realizar suas atividades cotidianas de vida diária.

Leal NFBS, et al. (2009) relata em seu estudo que a fisioterapia complexa descongestiva é a principal terapêutica para o linfedema, na qual os efeitos da FCD consistem na dilatação dos canais tissulares, proporcionando a formação de neoanastomoses linfáticas, estimulando os vasos linfáticos e motricidade dos linfangions com a melhora do fluxo e filtrando e renovando as células de defesa do organismo, eficácia do bombeamento muscular e articula, melhora da funcionalidade do membro, fortalecimento e prevenção de atrofia muscular, assim como redução da dor crônica em mulheres portadoras de linfedema.

Santos RO e Luz KRG (2017) apontam que a conduta que mais se faz eficaz para o tratamento do linfedema é a FCD, na qual tornou-se a mais adequada quando as modalidades de tratamento são realizadas em conjunto com técnicas combinadas de exercícios fisioterapêuticos linfocineticos e enfaixamento compressivo provocando reações fisiológicas beneficiam a paciente com redução do linfedema.

$\mathrm{Na}$ pesquisa realizada por Meirelles MCCC, et al. (2006) os resultados obtidos com o tratamento do linfedema através da terapia complexa descongestiva (TCD), na qual foi composta por técnicas como drenagem linfática manual, enfaixamento compressivo funcional, braçadeiras elásticas, exercícios, orientações de autocuidados e automassagem, destacaram resultados melhores e mais rápidos do que outros métodos não invasivos existentes.

Paz IA, et al. (2016) relata em seu estudo que: O tratamento do linfedema é de extrema importância para as pacientes acometidas, pois promove a desobstrução da rede linfática e proporciona melhora da questão emocional, destacando que ao contrário dos processos cirúrgicos, não apresenta efeitos adversos graves.

No estudo realizado por Haghighat $S$, et al. (2010), onde foi verificado a eficácia da TCD em relação a TCD Modificada (associada à compressão pneumática), em pacientes com linfedema de membro superior após cirurgia de câncer de mama, onde os resultados foram a promoção da redução no volume do membro afetado, porém, a redução foi mais significativa na aplicação da TCD isolada.

A terapia física complexa descongestiva quando incluindo exercícios, uso da eletroestimulação e drenagem linfática são métodos de extrema importância para evitar que o linfedema se instale e vire crônico, além de se obter um aumento e manutenção da amplitude de movimento na articulação do ombro homolateral à cirurgia (LUZ ND e LIMA ACG, 2011).

É visível que uma vez instalado, o linfedema pode ser controlado, porém, não há cura, podendo apenas ser reduzido significativamente logo após na primeira semana de tratamento, podendo, após a terceira semana, ocorrer uma redução menos significativa. A partir do momento que linfedema se instala, o tratamento deve ser continuado até a fase de manutenção da redução já conseguida anteriormente, contribuindo para reduzir a incidência de infecções e para melhora a qualidade de vida da paciente (LEAL NFBS, et al., 2009).

\section{CONSIDERAÇÕES FINAIS}

A fisioterapia enquanto ciência de cunho generalista, busca reestabelecer a funcionalidade do corpo humano, na atenção à saúde de pacientes portadores de linfedema de membro superior pós-mastectomia radical, buscando através dos recursos disponíveis a melhora da qualidade de vida desses pacientes. $O$ levantamento da pesquisa bibliográfica deixou evidente que a fisioterapia complexa descongestiva, composta por técnicas e recursos fisioterapêuticos que proporcionam aumento da funcionalidade, diminuição da dor, melhora da percepção corporal e melhora da qualidade de vida tem demonstrado grande eficácia na qual é a técnica mais recomendada para o tratamento de linfedema em mulheres pós-mastectomizadas. 


\section{REFERÊNCIAS}

1. ALBUQUERQUE VT, et al. Funcionalidade de membros superiores em mulheres após cirurgia para câncer de mama. 2013.

2. BRANDÃO ML, et al. Eficácia da terapia complexa descongestiva para linfedema nos membros inferiores: revisão sistemática. Jornal Vascular Brasileiro, 2020; 19.

3. CENDRON SW, et al. Fisioterapia complexa descongestiva associada a terapias de compressão no tratamento do linfedema secundário ao câncer de mama: uma revisão sistemática. Rev Bras Cancerol, 2015; 61: 49-58.

4. CORINTIO MN. Manual do aleitamento materno $3^{\circ}$ ed. Federação Brasileira das Associações de Ginecologia e Obstetrícia (FEBRASGO). 2015.

5. DE ROMA MAM, et al. Terapia Física Complexa no Linfedema em Pacientes Após Cirurgia de Câncer de Mama: Revisão Sistemática. Revista Pesquisa em Fisioterapia, 2016; 6(1).

6. DE SOUZA AS, NEVES PO. Complicações pós cirurgicas em mulheres submetidas à mastectomia. 2016. Reabilitação Física no Câncer de Mama, 1. ed. São Paulo: Roca LTDA, 2010.

7. HAGHIGHAT S, et al. Comparing two treatment methods for post mastectomy lymphedema: complex decongestive therapy alone and in combination with intermittent pneumatic compression. Lymphology, 2010; 43(1): 25-33.

8. LEAL NFBS, et al. Tratamentos fisioterapêuticos para o linfedema pós-câncer de mama: uma revisão de literatura. Revista LatinoAmericana de Enfermagem, 2009; 17(5).

9. LUZ ND, LIMA ACG. Recursos fisioterapêuticos em linfedema pós-mastectomia: uma revisão de literatura. Fisioterapia em Movimento, 2011; 24(1): 191-200.

10. MEIRELLES MCCC, et al. Avaliação de técnicas fisioterapêuticas no tratamento do Linfedema pós-cirurgia de mama em mulheres. Ribeirão Preto: USP, 2006; 7p.

11. NASCIMENTO SL, et al. Complicações e condutas fisioterapêuticas após cirurgia por câncer de mama: estudo retrospectivo, Rev. Fisioterapia e Pesquisa, 2012; 248- 255p.

12. NGOMANE AY, et al. Orientações domiciliares para mulheres em tratamento do câncer de mama. ConScientiae Saúde, 2014; 13(2): 196-202.

13. NOGUEIRA PVG. Efeitos da facilitação neuromuscular proprioceptiva na performance funcional de mulheres mastectomizadas. Fisioterapia Brasil, 2018; 6(1): 28-35.

14. PAIVA DMF, et al. Fatores associados ao linfedema em pacientes com câncer de mama. Revista Brasileira de Ginecologia e Obstetrícia, 2011; 33(2): 75-80.

15. PAZ IA, et al. Terapia complexa descongestiva no tratamento intensivo do linfedema: revisão sistemática. Fisioterapia e Pesquisa, 2016; 23(3): 311-317.

16. REZENDE LF, et al. Avaliação dos fatores de risco no linfedema pós-tratamento de câncer de mama. Jornal Vascular Brasileiro, 2010; 9(4): 233-238.

17. SANTIAGO GS, et al. Estudo comparativo do uso da drenagem linfática manual e terapia física complexa na reabilitação do linfedema de membro superior após tratamento cirúrgico do câncer de mama-revisão de literatura. 2016.

18. SANTOS DAD, et al. Atuação da fisioterapia no tratamento do linfedema após câncer de mama. Ensaios e Ciência: Ciências biológicas, agrárias e da saúde, 2010; 14(1): 177-186.

19. SANTOS RO, LUZ KRG. Fisioterapia Complexa Descongestiva no linfedema pós-mastectomia. Revista Ciência \& Saberes Facema, 2017; 2(4): 316-319.

20. SOARES HPS, et al. Terapia complexa descongestiva com uso de material alternativo na redução e controle do linfedema em pacientes de área endêmica de filariose: um ensaio clínico. Fisioterapia e Pesquisa, 2016; 23(3) 268277. 